\title{
Elasto-plastic damage modelling of beams and columns with mechanical degradation
}

\author{
R. Emre Erkmen*, Nadarajah Gowripalan ${ }^{\mathrm{a}}$ and Vute Sirivivatnanon ${ }^{\mathrm{b}}$ \\ School of Civil and Environmental Engineering, University of Technology Sydney, NSW 2007, Australia
}

(Received June 14, 2016, Revised November 24, 2016, Accepted January 2, 2017)

\begin{abstract}
Within the context of continuum mechanics, inelastic behaviours of constitutive responses are usually modelled by using phenomenological approaches. Elasto-plastic damage modelling is extensively used for concrete material in the case of progressive strength and stiffness deterioration. In this paper, a review of the main features of elasto-plastic damage modelling is presented for uniaxial stress-strain relationship. It has been reported in literature that the influence of Alkali-Silica Reaction (ASR) can lead to severe degradations in the modulus of elasticity and compression strength of the concrete material. In order to incorporate the effects of ASR related degradation, in this paper the constitutive model of concrete is based on the coupled damage-plasticity approach where degradation in concrete properties can be captured by adjusting the yield and damage criteria as well as the hardening moduli related parameters within the model. These parameters are adjusted according to results of concrete behaviour from the literature. The effect of ASR on the dynamic behaviour of a beam and a column are illustrated under moving load and cyclic load cases.
\end{abstract}

Keywords: alkali-slica reaction; coupled damage-plasticity; dynamic behaviour

\section{Introduction}

Alkali Silica Reaction (ASR) may significantly reduce the strength of concrete as illustrated by Swamy and AlAsali (1988). The strength reduction would lead to crack formations (Giaccio et al. 2008); it also degrades the modulus of elasticity (Multon et al. 2005) and causes excessive swelling which would lead to large deformations exceeding serviceability limit states; and the expansion of concrete generated by ASR develops stresses that leads to damage and degradation in strength. Therefore, ASR should be prevented when possible by selecting non-reactive aggregates. Where the use of reactive aggregates cannot be avoided, mitigative measures such as the use of supplementary cementitious materials and limitation of total alkali in concrete mixture must be implemented. However, structures already suffering from ASR require assessment. Thus, there is a need for accurate tools that are tailored for the analysis of ASR effects on structural behaviour. Such tools are required to capture the observed behaviour as well as to predict the long term effects and possible failure mechanisms for rehabilitation purposes.

Pietruszcak (1996) considered the effect of ASR on degradation of the concrete within the framework of elastoplasticity. However, the basic hypothesis of plasticity models that the elastic response remains the same in loading and unloading is no longer valid if the elastic response is

\footnotetext{
*Corresponding author, Lecturer

E-mail: emre.erkmen@uts.edu.au

${ }^{\text {a }}$ Senior Lecturer

${ }^{\mathrm{b}}$ Professor
}

Copyright $\odot 2017$ Techno-Press, Ltd.

http://www.techno-press.org/?journal=cac\&subpage $=8$ affected by the inelastic deformations. This kind of phenomena is observed in loading-unloading cycles of the concrete material, where the inelastic material pertains to cracks will also modify the elastic response. Therefore, damage modelling is also necessary to simulate the inelastic concrete behaviour under cyclic loading.

Coupled elasto-plastic and damage models have been applied extensively for the description of progressive failure of materials such as concrete, geomaterials, woods, steel and composites. Initial attempts of merging elasto-plastic and damage constitutive models can be found in Lemaitre (1985). Simo and Ju (1987) developed alternative strain and stress based formulations and algorithms for the coupled elasto-plastic damage constitutive modelling of materials and applied their theories for the simulation of the concrete material behaviour. Later, Ju (1989) developed an energybased coupled elasto-plastic damage modelling approach. Constitutive models that are capable of coupling elastoplasticity and damage were also used for plain concrete by Meschke et al. (1998), for concrete compaction by Herve et al. (2005) and for mild steel by Ayhan et al. (2013). Lee et al. (1998) introduced a plastic-damage model for concrete subjected to cyclic loading. Damage models have also been adopted for concrete by Pituba et al (2012) and Pituba (2015). Vaz and Owen (2001) developed an algorithm for failure predictions of multi-fracturing materials based on the elasto-plastic damage modelling approach. Al-Rub and Kim (2010) used coupled plasticity-damage model for the simulation of the fracture process of plain concrete. Jukic et al. (2014) incorporated embedded discontinuity approach in simulating the failure of reinforced concrete beams based on combined elasto-plastic damage modelling. Elastoplastic damage modelling approach has also been used for the modelling of concrete subjected to Alkali-Silica 
Reaction by Yingdi et al. (2014).

In this study, Euler-Bernoulli beam kinematic model is adopted and therefore stress-strain relationship is uniaxial. A review of the main features of elasto-plastic damage modelling is presented for uniaxial stress-strain relationship. The effects of ASR are incorporated into the stress strain relationship by adjusting the material model parameters according to Ibrahimbegovic et al. (2008). ASR effects are then considered as damage and its influence on the structural performance is illustrated in numerical studies. The steel material is assumed elasto-plastic.

\section{Coupled damage-plasticity model}

The uniaxial constitutive model of coupled damage and plasticity can be built on three basic hypotheses: additive decomposition of the total strain field, the strain energy and finally the plasticity and damage criteria.

- The first of them implies that the total deformation can be additively decomposed into elastic part $\varepsilon_{e}$, plastic part $\varepsilon_{p}$ and damage part $\varepsilon_{d}$, leading to $\varepsilon=\varepsilon_{e}+\varepsilon_{p}+\varepsilon_{d}$.

- The second ingredient of the model governing elastic response is specified in terms of strain energy. Assuming the simplest quadratic form in terms of the corresponding state variables we can write the strain energy as the sum of elastic, damage and plastic parts

$$
\begin{aligned}
& \Psi\left(\varepsilon, \varepsilon_{p}, \xi_{p}, \phi, \varepsilon_{d}, \xi_{d}\right)=\Psi^{e}\left(\varepsilon_{e}\right) \\
& +\Psi^{d}\left(\varepsilon_{d}, \phi\right)+\Xi^{p}\left(\xi_{p}\right)+\Xi^{d}\left(\xi_{d}\right)
\end{aligned}
$$

Where $\phi=\varphi /(1-\varphi)$ and $\varphi$ is the internal damage variable. Physically, damage in concrete is the result of the initiation, growth and coalescence of micro-cracks or micro-voids. Within the context of continuum mechanics, one may relate the degradation in material properties by introducing a scalar quantity if restricted to isotropic damage (Lemaitre 1985), i.e., $\varphi \in[0,1]$. In Eq. (1), hardening effects of the model can be accounted with variables $\xi_{p}$ for plasticity and $\xi_{d}$ for damage. Assuming linear isotropic hardening, the hardening functions for plasticity is $\Xi^{p}\left(\xi_{p}\right)=\frac{1}{2} K_{p} \xi_{p}{ }^{2}$ and the hardening functions for damage can be written as $\Xi^{d}\left(\xi_{p}\right)=\frac{1}{2} K_{d} \xi_{d}{ }^{2}$, where $K_{p}$ and $K_{d}$ are hardening moduli for plasticity and damage, respectively. Elastic strain energy can be written as $\Psi^{e}\left(\varepsilon_{e}\right)=\sigma \varepsilon_{e}-\chi^{e}$ in which $\chi^{e}(\sigma)=\frac{1}{2} E^{-1} \sigma^{2}$ is the complementary strain energy. The damage strain energy can be written as $\Psi^{d}\left(\varepsilon_{d}, \phi\right)=\sigma^{\mathrm{T}} \varepsilon_{d}-\chi^{d}(\sigma, \phi)$ in which $\chi^{d}(\sigma, \phi)=\frac{1}{2} E^{*} \varepsilon^{2}-\frac{1}{2} E^{-1} \sigma^{2}=\frac{1}{2} \phi E^{-1} \sigma^{2}$ was used and the reason for introducing $\Psi^{d}$ is to accommodate the degradation in the material stiffness due to inelastic deformations.

- The final group of basic model ingredients is provided to specify the elastic domain, where no change of internal variables takes place, along with the yield criteria and damage criteria as

$$
\begin{gathered}
\Phi^{p}\left(\sigma, q_{p}\right)=|\sigma|-\left(\sigma_{y}-q_{p}\right) \leq 0 \\
\Phi^{d}\left(\sigma, q_{d}\right)=|\sigma|-\left(\sigma_{f}-q_{d}\right) \leq 0
\end{gathered}
$$

Where $-q_{p}=\partial \Xi^{p}\left(\xi_{p}\right) / \partial \xi_{p}=K_{p} \xi_{p}$ and $-q_{d}=\partial \Xi^{p}\left(\xi_{d}\right) / \partial \xi_{d}=K_{d} \xi_{d}$ are conjugates to variables $\xi_{p}$ and $\xi_{d}$, respectively, $\sigma_{y}$ is the initial yield stress limit and $\sigma_{f}$ is the initial damage stress limit.

All necessary equations can be obtained from the above three sets of equations by the principles of maximum plastic and maximum damage dissipations. Firstly, we write the local form of second principle of thermodynamics. That is, the total inelastic dissipation is always non-negative, i.e., $\mathrm{d} \Omega=\sigma \mathrm{d} \varepsilon-\mathrm{d} \Psi \geq 0$. Subsequently, one obtains

$$
\begin{gathered}
\overbrace{\left(\sigma-\frac{\partial \Psi^{e}}{\partial \varepsilon_{e}}\right)}^{0} \mathrm{~d} \boldsymbol{\varepsilon}_{e}+\overbrace{\sigma \mathrm{d} \varepsilon_{p}-\frac{\partial \Xi^{p}\left(\xi_{p}\right)}{\xi_{p}} \mathrm{~d} \xi_{p}}^{\mathrm{d} \Omega^{p}}+ \\
\mathrm{d} \sigma \overbrace{\left(\frac{\partial \chi^{d}}{\partial \sigma}-\varepsilon_{d}\right)}^{0}+\overbrace{\frac{\partial \chi^{d}}{\partial \phi} \mathrm{d} \phi-\frac{\partial \Xi^{d}\left(\xi_{d}\right)}{\xi_{d}} \mathrm{~d} \xi_{d}}^{\mathrm{d} \Omega^{d}} \geq 0
\end{gathered}
$$

Where $\mathrm{d} \Omega^{p}$ and $\mathrm{d} \Omega^{d}$ are plastic and damage dissipation. From the above equation, the stress can be obtained from the elastic strain energy, i.e., $\sigma=\partial \Psi^{e} / \partial \varepsilon_{e}$. Note that in obtaining Eq. (4), $\sigma \mathrm{d} \varepsilon_{d}=\mathrm{d}\left(\sigma \varepsilon_{d}\right)-\mathrm{d} \sigma \varepsilon_{d}$ was used. The damage strain is defined through stress and the current value of damage compliance, i.e., $\varepsilon_{d}=\partial \chi^{d} / \partial \sigma=\phi E^{-1} \sigma$. Assuming that the stress constitutive equation and damage strain definition remain the same in an inelastic process we can conclude from dissipation inequality that

$$
\underbrace{\sigma \mathrm{d} \varepsilon_{p}+q_{p} \mathrm{~d} \xi_{p}}_{\text {plastic dissipation }}+\underbrace{\frac{1}{2} \mathrm{~d} \phi E^{-1} \sigma^{2}+q_{d} \mathrm{~d} \xi_{d}}_{\text {damage dissipation }} \geq 0
$$

\subsection{Plastic model}

Maximum plastic dissipation states that for a given plastic strain configuration $\varepsilon_{p}$ among all possible stress states $\tau$ satisfying the yield criterion (i.e., the ones on the yield surface), the actual stress states maximizes the plastic dissipation, $\quad \sigma \mathrm{d} \varepsilon_{p}+q_{p} \mathrm{~d} \xi_{p}=\max _{\Phi^{p}(\tau, p)=0}\left(\tau \mathrm{d} \varepsilon_{p}+p \mathrm{~d} \xi_{p}\right) . \quad$ The principle of maximum plastic dissipation implies normality in stress space considering that the yield surface is convex. In order to illustrate that, we use the Lagrange multiplier $\mathrm{d} \lambda_{p}$ and change of sign of the plastic dissipation to transform the constraint maximization problem to an unconstraint problem of minimization (Simo and Hughes 1998), i.e., $\quad L^{p}\left(\tau, p, \mathrm{~d} \lambda_{p}\right)=-\tau \mathrm{d} \varepsilon_{p}-p \mathrm{~d} \xi_{p}+\mathrm{d} \lambda_{p} \Phi^{p}(\tau, p) \geq 0$. This inequality is particularly useful in determining whether 
the constitutive relation of a material is thermodynamically allowable. This inequality is a statement concerning the irreversibility of natural processes, especially when energy dissipation is involved. The minimum of this function in stress space generates

$$
\begin{gathered}
\frac{\partial L^{p}\left(\sigma, q_{p}, \mathrm{~d} \lambda_{p}\right)}{\partial \sigma}=-\mathrm{d} \varepsilon_{p}+\mathrm{d} \lambda_{p} \frac{\partial \Phi^{p}\left(\sigma, q_{p}, \mathrm{~d} \lambda_{p}\right)}{\partial \sigma}=0 \\
\frac{\partial L^{p}\left(\sigma, q_{p}, \mathrm{~d} \lambda_{p}\right)}{\partial q_{p}}=-\mathrm{d} \xi_{p}+\mathrm{d} \lambda_{p} \frac{\partial \Phi^{p}\left(\sigma, q_{p}, \mathrm{~d} \lambda_{p}\right)}{\partial q_{p}}=0
\end{gathered}
$$

which can be interpreted as the evolution equations of the internal variables of the plastic model with Lagrange multiplier as the plastic multiplier. The minimization with respect to the Lagrange multiplier $d \lambda_{p}$ generates the yield condition, i.e., $\Phi^{p}\left(\sigma, q_{p}\right)=0$.

\subsubsection{Plastic flow rule}

This rule is inspired by the fact that during the loading which causes plastic deformations, the stresses should stay on the yield surface. Therefore, the stress increments should be tangential to the yield surface. Since the stress increments are produced by the elastic strain increments only, i.e., $\mathrm{d} \sigma=E d \varepsilon_{e}$, the directions of the elastic strains are also tangential to the yield surface. Since the plastic strain increments do not produce stresses, their directions should be normal to the yield surface. This normality criterion in Eq. (6) is also called the Prandtl-Reuss, where $d \lambda_{p}$ is the proportionality factor yet to be determined. Note that a negative proportionality factor $d \lambda_{p}$ would imply plastic unloading which cannot occur. There is only elastic unloading allowed in which case the proportionality factor $d \lambda_{p}$ is zero.

\subsubsection{Elasto-plastic tangent modulus}

From the consistency condition, when plastic flow occurs the stresses remain on the yield surface, i.e., $\mathrm{d} \Phi^{p}=\left(\partial \Phi^{p} / \partial \sigma\right) \mathrm{d} \sigma+\left(\partial \Phi^{p} / \partial q_{p}\right) \mathrm{d} q_{p}=0$.

By using $\mathrm{d} \sigma=E\left(\mathrm{~d} \varepsilon-\mathrm{d} \varepsilon_{p}-\mathrm{d} \varepsilon_{d}\right)$, and $\mathrm{d} \varepsilon_{p}=\mathrm{d} \lambda_{p} \operatorname{sign}(\sigma)$ in the equation above, the proportionality factor becomes $\mathrm{d} \lambda_{p}=\left(\mathrm{d} \varepsilon-\mathrm{d} \varepsilon_{d}\right) \operatorname{sign}(\sigma) E /\left(E+K_{p}\right)$.

For isotropic strain hardening under uniaxial stressstrain relationship $d \xi_{p}=d \lambda_{p}$ can be assumed (see Simo and Hughes 1998). Using the above equation for $d \lambda_{p}$ with $d \varepsilon_{p}=d \lambda_{p}$ and $q_{p}=-K_{p} \xi_{p}$ and substituting into $d \sigma=E\left(\mathrm{~d} \varepsilon-\mathrm{d} \varepsilon_{p}-d \varepsilon_{d}\right)$ produces

$$
\mathrm{d} \sigma=\left(E-\frac{E^{2}}{E+K_{p}}\right)\left(\mathrm{d} \varepsilon-\mathrm{d} \varepsilon_{d}\right)=C^{\mathrm{e} p}\left(\mathrm{~d} \varepsilon-\mathrm{d} \varepsilon_{d}\right)
$$

\subsection{Damage model}

\subsubsection{Effective stress concept}

The strain associated with a damaged state under the applied stress is equivalent to the strain associated with its fictitious undamaged state under effective stress. Using the hypothesis of strain equivalence it can be understood that the stress-strain relationship of damaged materials can be exchanged with the stress-strain relationship of the fictitious undamaged state but the Cauchy stress $\sigma$ should be replaced by the effective stress $\sigma^{*}$. For the practical damaged state, according to this hypothesis, the stress-strain relation can be written as $\varepsilon=E^{*-1} \sigma$. and in fictitious undamaged state, the stress strain relation can be written as $\varepsilon=E^{*-1} \sigma$. According to the concept of strain equivalence, we have $\sigma=E^{-1} E^{*} \sigma^{*}$ where $E^{-1} E^{*}$ an be considered as a function of stress transformation from the effective stress $\sigma^{*}$ to the Cauchy stress $\sigma$. For an isotropic damage state, the damaged modulus of elasticity can be obtained as $E^{*}=(1-\varphi) E$.

\subsubsection{Damage model derivations}

The damage model can be cast in an equivalent form similar to the one given for plasticity. In the case, where the damage model is activated and plasticity remains inactive, we can appeal to the principle of maximum damage dissipation to select among all admissible values of stress and hardening damage variables those which maximize the damage dissipation. Then, for a given damage strain configuration $\varepsilon_{d}$ among all possible stress states $\tau$ satisfying the damage criterion (i.e., the ones on the damage surface), the actual stress states maximizes the damage dissipation, $\sigma \mathrm{d} \varepsilon_{d}+q_{d} \mathrm{~d} \xi_{d}=\max _{\Phi^{d}(\tau, p)=0}\left(\tau \mathrm{d} \varepsilon_{d}+p \mathrm{~d} \xi_{d}\right)$. The principle of maximum damage dissipation implies normality in stress space considering that the damage surface is convex. In order to illustrate that, we use the Lagrange multiplier $d \lambda_{d}$ and change of sign of the damage dissipation to transform the constraint maximization problem to an unconstraint problem of minimization, i.e., $L^{d}\left(\tau, p, \mathrm{~d} \lambda_{d}\right)=-\tau \mathrm{d} \varepsilon_{d}-p \mathrm{~d} \xi_{d}+\mathrm{d} \lambda_{d} \Phi^{d}(\tau, p) \geq 0$. The minimum of this function in stress space generates

$$
\begin{aligned}
& \frac{\partial L^{d}\left(\sigma, q_{d}, \mathrm{~d} \lambda_{d}\right)}{\partial \sigma}=-E^{-1} \sigma \mathrm{d} \phi+\mathrm{d} \lambda_{d} \frac{\partial \Phi^{d}\left(\sigma, q_{d}, \mathrm{~d} \lambda_{d}\right)}{\partial \sigma}=0 \\
& \frac{\partial L^{d}\left(\sigma, q_{p}, \mathrm{~d} \lambda_{d}\right)}{\partial q_{d}}=-\mathrm{d} \xi_{d}+\mathrm{d} \lambda_{d} \frac{\partial \Phi^{d}\left(\sigma, q_{d}, \mathrm{~d} \lambda_{d}\right)}{\partial q_{d}}=0
\end{aligned}
$$

The minimization with respect to the Lagrange multiplier $d \lambda_{d}$ generates the damage condition, i.e., $\Phi^{d}(\sigma$, $\left.q_{d}\right)=0$.

\subsubsection{Elasto-plastic tangent modulus}

From the consistency condition one obtains,

$$
\mathrm{d} \Phi^{d}=\frac{\partial \Phi^{d}}{\partial \sigma} \mathrm{d} \sigma+\frac{\partial \Phi^{d}}{\partial q_{d}} \mathrm{~d} q_{d}=0
$$

By substituting Eq. (9) in $\mathrm{d} \sigma=\mathrm{d}\left(\phi^{-1} E \varepsilon_{d}\right)=\phi^{-1} E \mathrm{~d} \varepsilon_{d}+\varepsilon_{d} \mathrm{~d} \phi^{-1} E=\phi^{-1} E \mathrm{~d} \varepsilon_{d}-\phi^{-1} E \varepsilon_{d} \phi^{-1} \mathrm{~d} \phi$, one obtains $\mathrm{d} \sigma=\phi^{-1} E \mathrm{~d} \varepsilon_{d}-E \phi^{-1} \mathrm{~d} \lambda_{d} \operatorname{sign}(\sigma)$ with which, from $\mathrm{d} \xi_{d}=\mathrm{d} \lambda_{d}$ and $q_{d}=-K_{d} \xi_{d}$ by using in Eq. (11), $d \lambda_{d}$ 
becomes $\mathrm{d} \lambda_{d}=\mathrm{d} \varepsilon_{d} E \phi^{-1} \operatorname{sign}(\sigma) /\left(E \phi^{-1}+K_{d}\right)$. From which by substituting into $\mathrm{d} \sigma=\mathrm{d}\left(\phi^{-1} E \varepsilon_{d}\right)$ produces

$$
\mathrm{d} \sigma=\left(E \phi^{-1}-\frac{E^{2} \phi^{-2}}{E \phi^{-1}+K_{d}}\right) \mathrm{d} \varepsilon_{d}=K^{e d} \mathrm{~d} \varepsilon_{d}
$$

\subsubsection{Elasto-plastic damage coupling}

Finally for the case where both plasticity and damage models are active one can apply simultaneously the principle of maximum dissipation by equating Eqs. (8) and (12), and eliminating $d \varepsilon_{d}$ one obtains

$$
\mathrm{d} \sigma=K^{e d}\left(K^{e d}+C^{e p}\right)^{-1} C^{e p} \mathrm{~d} \varepsilon
$$

\section{Computational algorithm for stress update}

For numerical computation purposes procedures based on finite increments are needed. The central problem is to compute the internal variables which will provide an admissible stress field for a given strain at global iteration $i$ of step $n+1$, i.e., $\varepsilon_{n+1}^{i}$. This computation is accomplished by a local iterative scheme, with iteration counter denoted as $k$, where the current values of plastic and damage strain are known as $\varepsilon_{p}{ }^{k}$ and $\varepsilon_{d}{ }^{k}$. The corresponding stress field is $\sigma_{n+1}=E\left(\varepsilon_{n+1}^{i}-\varepsilon_{p}^{k}-\varepsilon_{d}^{k}\right)$. Computation will be initially carried out independently for the plasticity and damage part of the model, producing stress values $\sigma_{n+1}^{p}$ and $\sigma_{n+1}^{d}$. At the final stage the two stresses are forced to coincide which provides the converged values of plastic and damage deformations.

\subsection{Plastic computation}

Based on the given configuration $\left\{\sigma^{k}, \varepsilon^{k}, \varepsilon_{p}{ }^{k}, \xi_{p}{ }^{k}\right\}$ at $k^{\text {th }}$ iteration for a finite strain increment $\Delta \varepsilon^{k}$ and fixed damage strain $\varepsilon_{d}$, the problem is to determine the updated configuration $\left\{\sigma^{k+1}, \varepsilon^{k+1}, \varepsilon_{p}{ }^{k+1}, \xi_{p}{ }^{k+1}\right\}$ at $k+1$ considering the conditions of elasto-plastic deformations. Since the incremental integration is a strain driven process, the strain can be directly updated as $\varepsilon^{k+1}=\varepsilon^{k}+\Delta \varepsilon^{k}$. The updated stress, however, is dependent on the updated plastic strain, i.e., $\sigma^{k+1}=E\left(\varepsilon^{k+1}-\varepsilon_{p}{ }^{k+1}-\varepsilon_{d}\right)$. The plastic strain can be updated according to the finite incremental form of the flow rule as $\varepsilon_{p}{ }^{k+1}=\varepsilon_{p}{ }^{k}+\Delta \lambda_{\vec{k}} \operatorname{sign}\left(\sigma^{\tilde{k}}\right)$ in which $\Delta \lambda_{k}$ is incremental form of the slip rate or (the proportionality factor), which can also be used to update the hardening variable in the case of strain hardening, i.e., $\xi_{p}{ }^{k+1}=\xi_{p}{ }^{k}+\Delta \lambda_{\vec{k}}$. Once the incremental slip rate $\Delta \lambda_{k}$ is determined the updated configuration can be figured. In plasticity computations to obtain $\Delta \lambda_{k}$, we use the fact that the yield function stays as zero, i.e., $\Phi^{p}=0$ and $d \Phi^{p}=0$. Note that $\Phi^{p}=0$ can be written as $\Phi^{p}=\Phi^{p \text { trial }}-\Delta \lambda_{\tilde{k}}\left(E+K_{p}\right)=0$ based on which one obtains $\Delta \lambda_{\vec{k}}=\Phi^{\text {ptral }} /\left(E+K_{p}\right)$.

\subsection{Damage computation}

Based on the given configuration $\left\{\sigma^{l}, \varepsilon^{l}, \phi_{l}, \xi_{d}^{l}\right\}$ at $l^{\text {th }}$ iteration for a finite strain increment $\Delta \varepsilon^{l}$ the problem is to determine the updated configuration $\left\{\sigma^{l+1}, \varepsilon^{l+1}, \phi_{l+1}, \xi_{d}^{l+1}\right\}$ at $l+1$ considering the conditions of damage deformations. The trial stress is equal to the stress of the elasto-plastic device at the $l^{\text {th }}$ iteration, $\sigma^{\text {trial }}=\phi_{l}^{-1} E \varepsilon_{d}{ }^{l}$. From $\mathrm{d} \sigma=\phi^{-1} E \mathrm{~d} \varepsilon_{d}-E \phi^{-1} \mathrm{~d} \lambda_{d} \operatorname{sign}(\sigma)$ integrating numerically starting from the trial stress, one updates the stress based on the damage evolution as $\sigma^{l+1}=\sigma^{\text {trial }}-\phi^{-1} E \Delta \lambda_{l} \operatorname{sign}\left(\sigma^{\tilde{l}}\right)$. Note that the previous damage parameters $\phi_{l}$ have been used for calculating the trial stresses since the current parameters at $l+1$ is not known. Once the proportionality factor $\Delta \lambda_{l}$ is determined the updated configuration can be figured. The hardening variable can also be updated as $\xi_{d}^{l+1}=\xi_{d}^{l}+\Delta \lambda_{i}$ and the damage parameters can be updated as $\phi^{l+1}=\phi^{l}+\Delta \phi^{l}$, where $\Delta \phi=E \Delta \lambda_{d} /|\sigma|$. Note that $\Phi^{d}=0$ can be written as $\Phi^{d}=\Phi^{d \text { trial }}-\Delta \lambda_{l}\left(E \phi^{-1}+K_{d}\right)=0$. In that case $\Delta \lambda_{k}$ can be written as $\Delta \lambda_{l}=\Phi^{d \text { trial }} /\left(E \phi^{-1}+K_{d}\right)$.

\subsection{Coupling}

Damage and plasticity are evolving independently and they are parallel processes. The damage strain is an input in the plasticity calculations; however, two computational procedures can be advanced in parallel with no exchange of results. On the other hand, it is important to determine how they share the strain in a strain driven process and thus, what portion really is the damage strain. For this, the final results produced by two parts of the model should be compared against one another and any discrepancy or residual should be eliminated between the two stresses to satisfy equilibrium by adjusting the damage strain. Among those cases which satisfy elasto-plasticity for different damage strains the one that produces the same stress with the damage stress is the solution.

\section{Structural analysis procedure}

\subsection{Dynamic analysis}

In general the structural dynamic analysis will be implemented within the frame-work of finite element method and in an incremental iterative manner due to the nonlinearities involved in the problem. The dynamic equilibrium equations for the updated time $t+\Delta t$ can be written as

$$
\mathbf{R}(\mathbf{u})+\mathbf{C} \dot{\mathbf{u}}+\mathbf{M u ̈}=\mathbf{F}
$$


In which the first term $\mathbf{R}(\mathbf{u})$ is the internal stress resultants at the nodes and dependent on the current displacement configuration $\mathbf{u}, \mathbf{C}$ is a damping matrix, $\dot{\mathbf{u}}$ denotes time derivative of $\mathbf{u}(\equiv \mathrm{d} \mathbf{u} / \mathrm{d} t), \quad \mathbf{M}$ is the nodal equivalent mass matrix and $\mathbf{F}$ is the external load vector. The incremental form of the nonlinear equilibrium equations can be obtained by subtracting the virtual work expressions of two adjacent equilibrium states and then linearizing the result by omitting the second and higher order terms as

$$
\mathbf{K}_{T} \delta \mathbf{u}+\mathbf{C} \delta \dot{\mathbf{u}}+\mathbf{M} \delta \ddot{\mathbf{u}}-\delta \mathbf{F}=0
$$

In which $\delta \mathbf{u}, \delta \dot{\mathbf{u}}$, and $\delta \ddot{\mathbf{u}}$ are incremental displacement, incremental velocity and incremental acceleration vectors respectively and $\delta \mathbf{F}$ is the incremental time-dependent load and $\mathbf{K}_{T}$ is the tangent stiffness matrix, constant acceleration method of Newmark (1959) is implemented to obtain a step by step numerical solution, based on which the dynamic equilibrium equation can be written as

$$
\delta \mathbf{F}_{e f f}=\mathbf{K}_{e f f} \delta \mathbf{u}_{t}
$$

in which $\delta \mathbf{F}_{\text {eff }}$ is the effective incremental load vector and $\mathbf{K}_{\text {eff }}$ is the effective stiffness matrix given by

$$
\delta \mathbf{F}_{\text {eff }}=\delta \mathbf{F}+\mathbf{M}\left(\frac{4}{\delta t} \dot{\mathbf{u}}_{t}+2 \ddot{\mathbf{u}}_{t}\right)+2 \mathbf{C} \dot{\mathbf{u}}_{t}
$$

and

$$
\mathbf{K}_{e f f}=\mathbf{K}_{T}+\frac{2}{\delta t} \mathbf{C}+\frac{4}{\delta t^{2}} \mathbf{M}
$$

In Eqs. (17) and (18), $\ddot{\mathbf{u}}_{t}$ and $\dot{\mathbf{u}}_{t}$ are the acceleration and velocity vectors at the previous time step and $\delta t$ is the prescribed time step increment. By assuming that the acceleration, velocity and displacement vectors at the previous time step are known, and using the solution of Eq. (16), the incremental acceleration and velocity vectors can be obtained as

$$
\delta \ddot{\mathbf{u}}_{t}=\frac{4}{\delta t^{2}} \delta \mathbf{u}_{t}-\frac{4}{\delta t} \dot{\mathbf{u}}_{t}-2 \ddot{\mathbf{u}}_{t} \text { and } \delta \dot{\mathbf{u}}_{t}=\frac{2}{\delta t} \delta \mathbf{u}_{t}-2 \dot{\mathbf{u}}_{t}
$$

and the displacement, velocity and acceleration vectors for the current time step can be obtained as

$$
\mathbf{u}_{t+\delta t}=\mathbf{u}_{t}+\delta \mathbf{u}_{t}, \quad \dot{\mathbf{u}}_{t+\delta t}=\dot{\mathbf{u}}_{t}+\delta \dot{\mathbf{u}}_{t} \text { and } \ddot{\mathbf{u}}_{t+\delta t}=\ddot{\mathbf{u}}_{t}+\delta \ddot{\mathbf{u}}_{t}
$$

Since the tangent stiffness matrix $K_{T}$ is only an approximation to the real stiffness of the structure for a time step $\delta t$, an unbalanced force vector $\delta \mathbf{U}_{t+\delta t}^{j}$ exists at the $j^{\text {th }}$ iteration. This unbalanced force can be calculated from the nonlinear equilibrium at the current time step from

$$
\delta \mathbf{U}_{t+\delta t}^{j}=\mathbf{F}(t+\delta t)-\left[\mathbf{R}\left(\mathbf{u}_{t+\delta t}^{j}\right)+\mathbf{C} \dot{\mathbf{u}}_{t+\delta t}^{j}+\mathbf{M} \ddot{\mathbf{u}}_{t+\delta t}^{j}\right]
$$

The unbalanced force can be introduced into the incremental equilibrium equation, so that

$$
\delta \mathbf{U}_{t+\delta t}^{j}+\delta \mathbf{F}_{\text {eff }}=\mathbf{K}_{\text {eff }} \delta \mathbf{u}_{t}^{j}
$$

and an iterative process can be adopted over the time step $\delta t$ by recalculating the unbalanced force $\delta \mathbf{U}_{t+\delta t}^{j}$ for the updated displacement $\mathbf{u}_{t+\delta t}^{j}$, velocity $\dot{\mathbf{u}}_{t+\delta t}^{j}$ and acceleration $\ddot{\mathbf{u}}_{t+\delta t}^{j}$ vectors. The unbalanced force vector $\delta \mathbf{U}_{t+\delta t}^{j}$ is then added into the incremental equilibrium for the next $j^{\text {th }}$ iteration. A criterion for the error in a selected norm, e.g.,

$$
\frac{\max \left|\delta \mathbf{u}_{t}^{j}\right|}{\sum_{j=1}^{n} \max \left|\delta \mathbf{u}_{t}^{j}\right|}<\varepsilon_{t o l}
$$

for the incremental displacement vector $\delta \mathbf{u}_{t}$ can be assigned to terminate the loop for $j$ and to move on to the next time step for a predetermined tolerance $\varepsilon_{t o l}$. The stress resultant vector $\mathbf{R}\left(\mathbf{u}_{t+\delta t}^{j}\right)$ at the $j^{\text {th }}$ iteration of the current time step $t+\delta t$ in Eq. (21) is calculated from section 3 by using numerical integration at the selected integration points.

\subsection{Static analysis}

Static analysis procedure can be obtained as a special case from Eq. (14) by removing the inertia and damping effects. In order to have a displacement control algorithm to be able trace the post peak values in load-displacement curve the incremental form of the equilibrium in Eq. (15) is replaced with

$$
\mathbf{K}_{i}^{j} \delta \mathbf{u}_{i}^{j}=\mathbf{r}_{i}^{j}+\delta \lambda_{i}^{j} \mathbf{F}
$$

where $j$ indicates the iteration number within the $i^{\text {th }}$ incremental step and $\mathbf{r}_{i}^{j}$ is the residual force vector, which is the difference between the internal and external forces, i.e., $\mathbf{r}_{i}^{j}=\lambda_{i}^{j} \mathbf{F}-\mathbf{R}\left(\mathbf{u}_{i}^{j}\right)$ where $\lambda_{i}^{j}$ is a load control parameter and $F$ is the applied load vector. The control parameter is adjusted in order to obtain a prescribed displacement at a selected point. Details of the displacement update procedure and the adjustment of the control parameter can be found in several references (e.g., Batoz and Dhatt 1979).

\section{Case studies}

\subsection{Adjustment of the parameters of the concrete} model

Firstly the parameters of the elasto-plastic damage model for concrete are adjusted to fit the concrete model employed by Ibrahimbegovic et al. (2008) which has been validated by comparisons with experimental results. As 


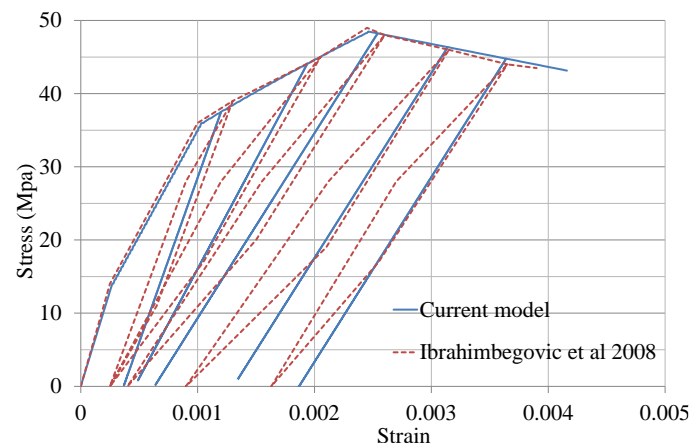

Fig. 1 Numerical results for cyclic behaviour of concrete in compression

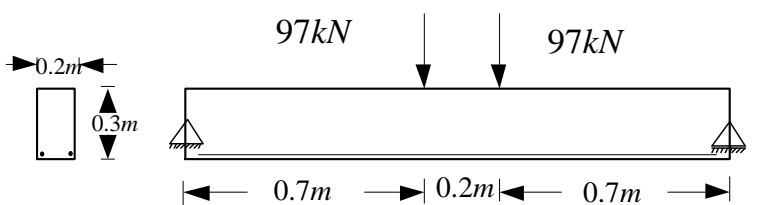

Fig. 2 A simply supported beam tested by Takahashi et al. (1997)

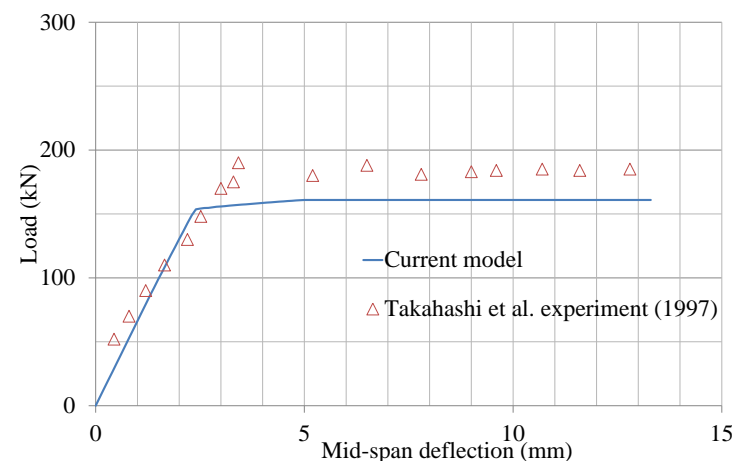

Fig. 3 Load vs mid-span deflection results for the tested beam by Takahashi et al. (1997)

shown in Fig. 1, there is very good agreement between the current model and the concrete model of Ibrahimbegovic et al. (2008). Following material properties for concrete are chosen; Young's modulus $E=52 \mathrm{GPa}, \sigma_{y}=14 \mathrm{MPa}, \sigma_{f}=36$ $\mathrm{MPa}, \sigma_{u}=48 \mathrm{MPa}, K_{p}=65 \mathrm{GPa}, K_{d}=14.5 \mathrm{GPa}$ and the hardening modulus after ultimate stress were taken as $K_{p}=-$ $2.8 \mathrm{GPa}$ and $K_{d}=-1.6 \mathrm{GPa}$. In all examples the same hardening parameters are adopted.

\subsection{Comparison with the beam experiment of Takahashi et al. (1997)}

The test of Takahashi et al. (1997) is examined in this example. The tested beam has a $1.6 \mathrm{~m}$ span and $200 \times 300$ $\mathrm{mm}^{2}$ cross-section as shown in the Fig. 2 .

The Young's modulus for concrete is $E_{c}=44.5 \mathrm{Gpa}$ and the ultimate compressive strength of concrete is $40.3 \mathrm{MPa}$. Accordingly, the yield stress is taken as $\sigma_{y}=14 \mathrm{MPa}$ and the fracture stress is taken as $\sigma_{f}=36 \mathrm{MPa}$. The tensile strength capacity has been assumed zero. Total of $593 \mathrm{~mm}^{2}$ tensile reinforcement has been used. Steel reinforcement has the modulus of elasticity $E_{s}=180 \mathrm{Gpa}$ and yield strength of $\sigma_{y}=371 \mathrm{MPa}$. It should also be noted that steel has assumed
Table 1 Mechanical property as percentage of the value (Gowripalan et al. 2016)

\begin{tabular}{ccc}
\hline \hline Property & \multicolumn{2}{c}{ Percentage } \\
\hline Uniaxial Compressive strength & 60 & 60 \\
Modulus of Elasticity & 50 & 35 \\
\hline
\end{tabular}

Table 2 Calculation of the damage parameter due to ASR related reduction in Modulus of Elasticity

\begin{tabular}{ccc}
\hline \hline Property & \multicolumn{2}{c}{ Percentage } \\
\hline Modulus of Elasticity & 50 & 35 \\
\hline$\varphi=1-E^{*} / E$ & $1-0.5=0.5$ & $1-0.35=0.65$ \\
$\phi=\varphi /(1-\varphi)$ & $0.5 /(1-0.5)=1$ & $0.65 /(1-0.65)=1.857$ \\
\hline
\end{tabular}

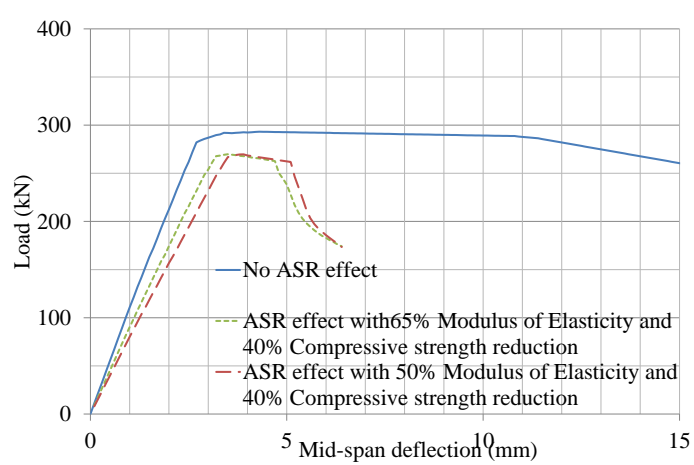

Fig. 4 Effect of ASR on the Load-Deflection behaviour of beams

to have zero hardening modulus. Concrete cover is assumed to be $50 \mathrm{~mm}$.

As shown in Fig. 3, the load to mid-span deflection results of the developed numerical analysis are in very good agreement with those of the experimental results of Takahashi. The load value corresponding to $12.8 \mathrm{~mm}$ deflection in Takashi experiment is $185 \mathrm{kN}$ while in the current model the corresponding load value is $161 \mathrm{kN}$.

\subsection{Effect of ASR on the nonlinear static analysis} results of a beam

In this example the effect of ASR on the structural behaviour is illustrated. In Table 1 we summarize the effect at two different stages of ASR reaction.

Damage can be defined as the impairment of the stress transmitting capacity as a result of the presence of microcracks. ASR is causing damage as its effect is irreversible and it degrades the material. Therefore, within the elasto-plastic damage modelling framework damage parameter is adjusted for both cases to introduce the ASR effect on the modulus of elasticity.

On the other hand, reduction in the ultimate stresses to $60 \%$ of its original capacity is also introduced due to ASR effect. The same beam shown in Fig. 2 is analysed however, only the reinforcement is increased to $550 \mathrm{~mm}^{2}$ each instead of $296.5 \mathrm{~mm}^{2}$ in the beam experiment of Takahashi et al. (1997). As can be seen from Fig. 4 that this amount of reinforcement is based on a balanced design where the failure of the beam is ductile. The effect of ASR is also 


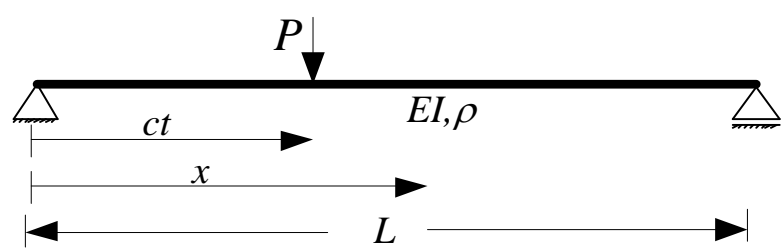

Fig. 5 Moving load on a simply supported beam

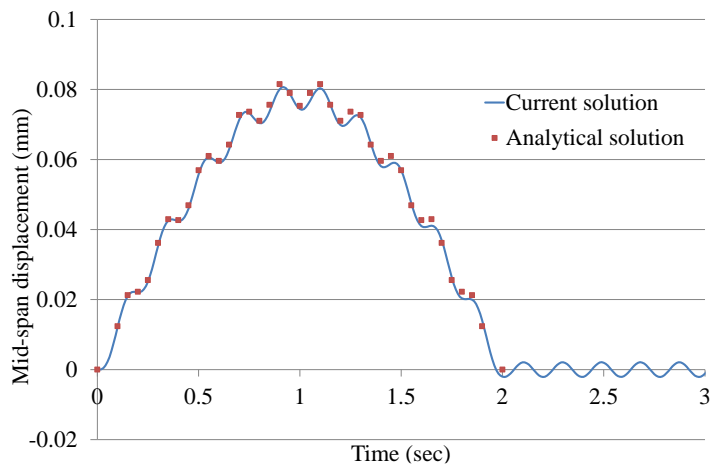

Fig. 6 Comparison of the analytical solution with the numerical solution

illustrated in the figure as there is a reduction in the ultimate load capacity and significant reduction in the ductility of the beam. It should be noted that these significant loses in mechanical properties do not all occur at the same rate (e.g., Swamy and Al-Asali 1988). In real structures it may take tens of years to have significant degradation in the material properties due to ASR. However, the degradation process may be accelerated in experiments by introducing excessive heat and humidity in to the environment.

As shown in Fig. 4, ASR effect can cause around 9\% reduction in the ultimate load carrying capacity as the peak point of the load reduces from $293 \mathrm{kN}$ down to approximately $269 \mathrm{kN}$. There is also significant reduction in the ductility of the beam due to early crushing of the concrete.

\subsection{Effect of ASR on the linear dynamic analysis results of a beam}

In this example, the dynamic analysis procedure is tested by comparison with the analytical solution. As shown in Fig. 5, a load $P$ is moving at a constant velocity $c$ on a simply supported beam which has a weight per length of $\rho$ and rigidity per length $E I$. The moving load is sufficiently small so that the material stays within the elastic limit both in tension and compression.

The analytical solution for time dependent deflection function at position $x$ at time $t$ can be written as (Olsson 1991)

$$
v(x, t)=\frac{P L^{3}}{48 E I} \sum_{j=1}^{\infty} \sin \left(\frac{j \pi x}{L}\right) \frac{1}{j^{2}\left(j^{2}-\alpha^{2}\right)}\left[\sin \left(\frac{j \pi c t}{L}\right)-\frac{\alpha}{j} \sin \left(\omega_{j} t\right)\right]
$$

in which $\omega_{j}^{2}=\frac{j^{4} \pi^{4}}{L^{4}} \frac{E I}{\rho}$ and $\alpha=\frac{L}{\pi} \sqrt{\frac{\rho}{E I}}$.

The moving load is $P=1 \mathrm{kN}$, the velocity is $c=10 \mathrm{~m} / \mathrm{sec}$, the weight per length is $\rho=0.0012 \mathrm{~N} / \mathrm{mm}$, rigidity is

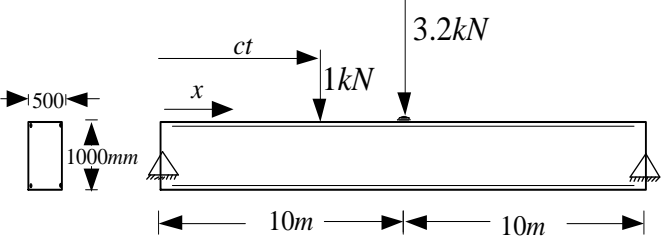

Fig. 7 Beam under moving load

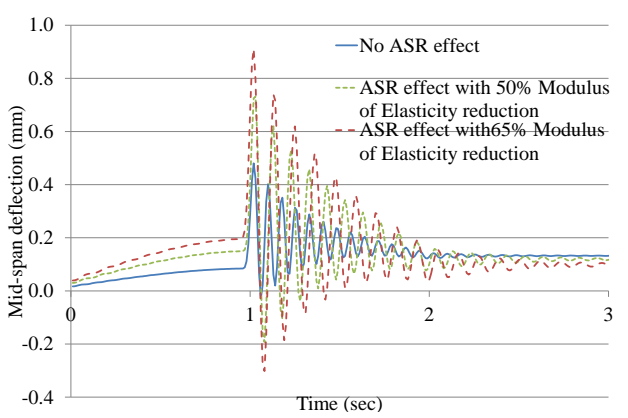

Fig. 8 Moving load analysis results of the beam

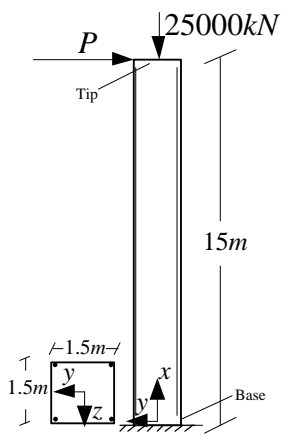

Fig. 9 Pier under cyclic load

$E I=2.145 ? 10^{15} \mathrm{Nmm}^{2}$ and the beam span is $L=20 \mathrm{~m}$. No damping is assumed. Comparisons of results based on the analytical and the proposed numerical solutions are shown in Fig. 6 below.

As shown in Fig. 6 the results are in perfect agreement and thus the numerical solution procedure for dynamic loading is validated. The same beam, with the dimensions as shown in Fig. 7 below, is analysed under moving load plus a sudden impact load of $3.2 \mathrm{kN}$ introduced at $1 \mathrm{sec}$ to simulate the effect of a speed bump at the mid-span. Low damping values are typical of most practical structures and damping ratio of $1 \%$ of the stiffness is used in this example. The material stays elastic during the analysis.

Three types of analysis are compared as shown in Fig. 8, i.e., beam without the ASR effect and two different beams with the ASR effect. The deflections significantly increase when the reduction of the elasticity modulus due to ASR is considered as given in Table 1.

For the case with no ASR effect, the maximum deflection at around $1 \mathrm{sec}$ is $0.48 \mathrm{~mm}$, whereas the maximum deflections corresponding to $65 \%$ and $50 \%$ modulus of elasticity reductions are $0.91 \mathrm{~mm}$ and $0.73 \mathrm{~mm}$, respectively.

5.5 Effect of ASR on the nonlinear dynamic analysis results of a column 


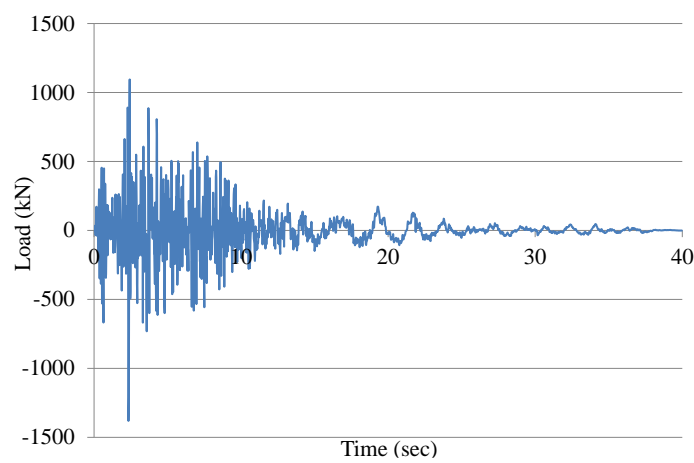

Fig. 10 Applied earthquake load based on Northridge 1994

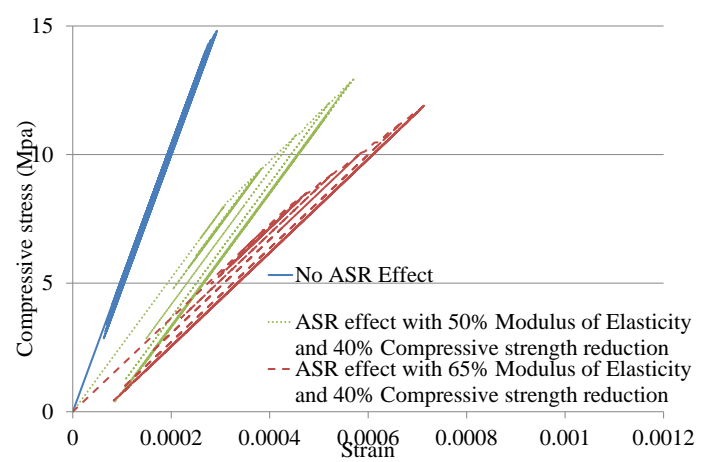

Fig. 11 Compressive stress-strain relationship at the root of the column

The fixed-base-free-end bridge pier shown in Fig. 9 is considered to be under $25 \times 10^{3} \mathrm{kN}$ vertical load. At $x=15 \mathrm{~m}$, $y=0, z=0$, the pier is subjected to the Earthquake load as shown in Fig. 10. The earthquake data is taken from the Northridge 1994 recordings (PEER Strong-Motion Database http:// peer.berkeley.edu).

Damping ratio is taken as $0.25 \%$ of the stiffness for the analysis. There is $6 \%$ reinforcement with the elasticity modulus of $200 \mathrm{GPa}$ and yield strength of $350 \mathrm{MPa}$ with no strain hardening. Comparisons of the 3 cases are illustrated in Fig. 11. The first case has no ASR effect and concrete properties have been taken as $E=52 \mathrm{GPa}, \sigma_{y}=14 \mathrm{MPa}, \sigma_{f}=36$ $\mathrm{MPa}, \sigma_{u}=48 \mathrm{MPa}$. When ASR effect is considered according to Table 1, the concrete properties have been dropped to $\sigma_{y}=8.2 \mathrm{MPa}, \sigma_{f}=21.3 \mathrm{MPa}, \sigma_{u}=30 \mathrm{MPa}$ due to $40 \%$ reduction in compressive strength of concrete. Concrete cover for reinforcement is $50 \mathrm{~mm}$. The ASR effect on the modulus of elasticity is considered by using the damage parameter $f$ and calculated as in Table 2 .

In this example the material reaches beyond the elastic limit. For example at the fibre of coordinates $x=0, y=0.75$ $\mathrm{m}, \quad z=0$ the compressive stress-strain relationship of concrete for all three cases can be obtained as shown in Fig. 11.

As shown in Fig. 12, the effect of ASR causes significant difference in the predictions of deflections compared to the case with no ASR.

For the case with no ASR effect, the maximum deflection during the first 40 seconds is $12.8 \mathrm{~mm}$, whereas the maximum deflections corresponding to $65 \%$ and $50 \%$ modulus of elasticity reductions are $35 \mathrm{~mm}$ and $26.9 \mathrm{~mm}$, respectively.

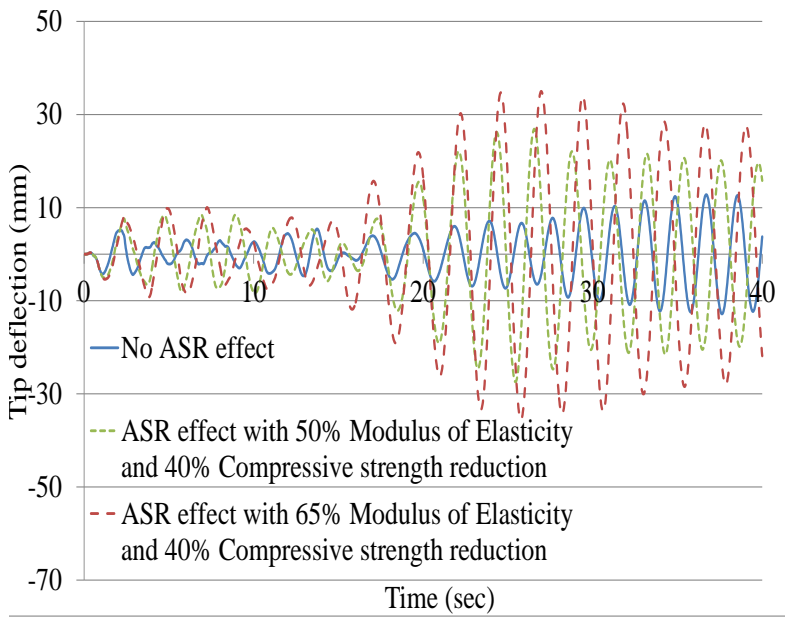

Fig. 12 Tip deflection results in time

\section{Conclusions}

In this paper, the behaviour of concrete is modelled within the elasto-plastic damage framework. Reduction in modulus of elasticity due to ASR effects is considered as damage effect. A procedure for dynamic analysis is developed to be able to capture cyclic load effects and validated by comparisons with a known solution. The influence of ASR related reductions in the modulus of elasticity and compressive strength on the structural behaviour is illustrated throughout the selected examples. It has been shown that ASR effects can cause significant reduction in the ductility of beams. There can also be significant increase in the deformations due to ASR related reduction of material properties.

\section{Acknowledgments}

This research is funded through an Australian Research Council Research Hub for Nanoscience Based Construction Materials Manufacturing (NANOCOMM) with the support of the Cement Concrete and Aggregates Australia (CCAA). The authors are grateful for the financial support of the Australian Research Council (IH150100006) in conducting this study.

\section{References}

Al-Rub, R.K.A. and Kim, S.M. (2010), "Computational applications of coupled plasticity-damage constitutive model for simulating plain concrete fracture", Eng. Fract. Mech., 77(10), 1577-1603.

Ayhan, B., Jehel, P., Brancherie, D. and Ibrahimbegovic, A. (2013), "Coupled damage-plasticity model for cyclic loading: Theoretical formulation and numerical implementation", Eng. Struct., 50, 30-42.

Batoz, J.L. and Dhatt, G. (1979), "Incremental displacement algorithms for displacement problems", J. Numer. Meth. Eng., 14(8), 1262-1267.

Giaccio, G., Zerbino, R., Ponce, J.M. and Batic, O.R. (2008), "Mechanical behaviour of concretes damaged by alkali-silica 
reaction", Cement Concrete Res., 38(7), 993-1004.

Gowripalan, N., Erkmen, R.E., Nejadi, S. and Sirivivatnanon, V. (2016), "Effects of Alkali-silica reaction on structural performance", University of Technology Sydney-Research Report.

Herve, G., Gatuingt, F. and Ibrahimbegovic, A. (2005), “On numerical implementation of acoupled rate dependent damageplasticity constitutive model for concrete in application to highrate dynamics", Eng. Comput., 22(5-6), 381-405.

Ibrahimbegovic, A., Jehel, P. and Davenne, L. (2008), "Coupled damage-plasticity constitutive and direct stress interpolation", Comput. Mech., 42(1), 1-11.

Ju, J.W. (1989), "On energy-based coupled elasto-plastic damage theories: Constitutive modelling and computational aspects", $J$. Sol. Struct., 25(7), 803-833.

Jukic, M., Brank, B. and Ibrahimbegovic, A. (2014), "Failure analysis of reinforced concrete frames by beam finite element that combines damage, plasticity and embedded discontinuity", Eng. Struct., 75, 507-527.

Lee, J. and Fenves, G.L. (1998), "Plastic-damage model for cyclic loading of concrete", J. Eng. Mech., 124(8), 892-900.

Lemaitre, J. (1985), "A continuous damage mechanics model for ductile fracture", J. Eng. Mater. Technol., 107(1), 83-89.

Lemaitre, J. (1985), "Coupled elasto-plasticity and damage constitutive equations", Comput. Meth. Appl. Mech. Eng., 51(13), 31-49.

Lemaitre, J. and Desmorat, R. (2005), Engineering Damage Mechanics, Springer.

Liao, Y., Chen, D., Liu, Z., Ouyang, F. and Hou, L. (2014), "Elastoplastic-damage compression constitutive model for cementitious material subjected to alkali-silica reaction", J. Adv. Concrete Technol., 12(5), 158-166.

Meschke, G., Lackner, R. and Mang, H. (1998), "An anisotropic elastoplastic-damage model forplain concrete", J. Numer. Meth. Eng., 42(4), 703-727

Multon, S., Seignol, J.F. and Toutlemoonde, F. (2005), "Structural behaviour of concrete beams affected by alkali-silica reaction", ACI Mater. J., 102(2), 67-76.

Newmark, N.M. (1959), "A method of computation for structural dynamics", J. Eng. Mech. Div., 85(3), 67-94.

Olsson, M. (1991), "On the fundamental moving load problem", $J$. Sound Vibr., 145(2), 299-307.

Pietruszczak, S (1996), "On the mechanical behaviour of concrete subject to alkali silica reaction", Comput. Struct., 58, 10931097.

Pituba, J.C. (2015), "A damage model formulation: Unilateral effect and RC structures analysis", Comput. Concrete, 15(5), 709-733.

Pituba, J.C. and Delalibera, R.G. (2012), "Numerical and statistical analysis about displacements in reinforced concrete beams using damage mechanics", Comput. Concrete, 10(3), 307-330.

Simo, J.C. and Hughes, T.J.R. (1998), Computational Inelasticity, Springer.

Simo, J.C. and Ju, J.W. (1987), "Strain- and stress-based continuum damage models-I. formulation", J. Sol. Struct., 23, 821-840

Simo, J.C. and Ju, J.W. (1987), "Strain- and stress-based continuum damage models-II. computational aspects", J. Sol. Struct., 23(7), 841-869.

Swamy, R.N. and Al-Asali, M.M. (1988), "Engineering properties of concrete affected by alkali-silica reaction", ACI Mater. J., 85(5), 367-374.

Takahashi, Y., Sato, Y., Ueda, T., Maeda, T. and Kobayashi, A. (1997), "Flexural behaviour of RC beams with externally bonded carbon fiber sheet", Proceedings of the $3 \mathrm{rd}$ International Symposium on on-metalic (FRP) Reinforced concrete Structures.

Vaz, M. and Owen, D.R.J. (1996), "Aspects of ductile fracture and adaptive mesh refinement in damaged elasto-plastic materials", J. Numer. Meth. Eng., 50(1), 29-54.

$C C$ 